\title{
Síntese de pós de alumina por meio de um método Pechini modificado com adição de sementes em diferentes atmosferas de calcinação
}

\section{(Synthesis of alumina powders by a modified Pechini method with addition of seeds in different calcination atmospheres)}

\author{
R. E. P. Salem ${ }^{1}$, A. S. A. Chinelatto ${ }^{2}$, A. L. Chinelatto $^{3}$ \\ ${ }^{l}$ Coordenação de Engenharia de Materiais, Universidade Tecnológica Federal do Paraná, Campus Londrina, \\ Av. dos Pioneiros 3131, Londrina, PR 86036-360 \\ ${ }^{2}$ Departamento de Engenharia de Materiais, Universidade Estadual de Ponta Grossa, Av. Gal. Carlos \\ Cavalcanti 4748, Ponta Grossa, PR 84030-900 \\ raphaelsalem@utfpr.edu.br,adriana@uepg.br,adilson@uepg.br
}

\begin{abstract}
Resumo
A alumina é um material muito estudado devido às suas excelentes características refratárias e propriedades mecânicas. A fase $\alpha$, mais estável, tem uma temperatura de formação de $\sim 1200^{\circ} \mathrm{C}$. Devido à sua elevada temperatura de formação, muitas pesquisas vêm buscando sua redução através da adição de sementes (seeds) da fase $\alpha$ em processos químicos de síntese. Este trabalho teve como objetivo sintetizar pós de $\alpha$-alumina pelo método Pechini com adição de sementes e comparar a calcinação ao ar e com um fluxo passante de $\mathrm{O}_{2}$, verificando sua influência nas características dos pós obtidos. Tais pós foram caracterizados por difração de raios $\mathrm{X}$, espectroscopia no infravermelho e microscopia eletrônica de varredura. Os resultados foram comparados com os dos mesmos pós calcinados ao ar. A adição de sementes favoreceu a diminuição da temperatura de transformação em fase $\alpha$, sendo que esta já é identificada a $1000{ }^{\circ} \mathrm{C}$. A presença de oxigênio na atmosfera de calcinação também favoreceu a formação de fase $\alpha$ a $900{ }^{\circ} \mathrm{C}$, temperatura inferior à dos pós calcinados ao ar.
\end{abstract}

Palavras-chave: síntese, Pechini, alumina, seeds, oxigênio.

Abstract

Alumina is a largely studied material due to its excellent refractory characteristics and mechanical properties. The $\alpha$-phase, the most stable one, has a formation temperature of about $1200^{\circ} \mathrm{C}$. Due to this high temperature, many researchers have been trying to decrease this temperature by addition of seeds of $\alpha$-phase in chemical processes of synthesis. This work had the purpose to synthesize powders of $\alpha$-alumina by the Pechini method with seeding and to compare calcination under air and with a $\mathrm{O}_{2}$ flow, thus verifying its influence on the characteristics of the obtained powders. The powders were characterized by X-ray diffraction, infrared spectroscopy and scanning electron microscopy. The results were compared with those of the same powders calcined under air. The seeding favored a decrease in the temperature of phase transformation to $\alpha$-phase, since it is identified in the powders calcined at $1000{ }^{\circ} \mathrm{C}$. The presence of oxygen in the calcination atmosphere also led to the formation of the $\alpha$-phase at $900{ }^{\circ} \mathrm{C}$, temperature lower than those of the powders calcined under air.

Keywords: synthesis, Pechini, alumina, seeds, oxygen.

\section{INTRODUÇÃO}

A fase $\alpha$, a mais estável da alumina, tem uma variedade muito grande de aplicações, principalmente no que concerne às suas propriedades mecânicas, térmicas e elétricas [1]. No processo industrial mais utilizado para produção da alumina, o processo Bayer, no qual se obtém a alumina a partir da bauxita, são necessárias temperaturas da ordem de $1200{ }^{\circ} \mathrm{C}$ para transformar completamente as aluminas de transição na fase $\alpha$. Este processo gera um pó agregado, de grande tamanho de partícula, que necessita passar por etapas subseqüentes de moagem para ser utilizada [2]. O óxido de alumínio é conhecido por existir em diversas formas polimórficas metaestáveis antes da transformação completa para a fase $\alpha$, que é termodinamicamente estável. Estes polimorfos incluem $\gamma$ (gama), $\theta$ (teta), $\eta$ (eta), $\delta$ (delta), $\chi$ (chi), $\kappa$ (kappa) e $\beta$ (beta) alumina. A formação de $\alpha$-alumina a partir da $\gamma$-alumina ocorre, durante o aquecimento, através da série de transformações polimórficas: $\gamma$-alumina $\rightarrow \delta$ alumina $\rightarrow \theta$-alumina $\rightarrow \alpha$-alumina, com a formação de $\alpha$-aluminaem temperaturas próximas a $1200{ }^{\circ} \mathrm{C}$. As transformações $\gamma \rightarrow \delta$ e $\delta \rightarrow \theta$ são de deslocamento, com energias de ativação relativamente baixas. A transformação $\theta \rightarrow \alpha$ é reconstrutiva e ocorre através de processo de nucleação e crescimento [3]. Visto que a maior parte da energia de ativação é requerida para o processo de nucleação, são necessárias elevadas temperaturas para nuclear $\alpha$-alumina, normalmente na faixa de $1200{ }^{\circ} \mathrm{C}$. 
Uma alta temperatura de transformação resulta sempre na formação de partículas mais grosseiras e formação de aglomerados fortes no pó [3]. A formação de uma, duas ou mais aluminas de transição antes da transformação completa para a fase estável $\alpha$-alumina depende das condições de processamento, do grau de cristalinidade e da presença de impurezas nos materiais de partida [3-7]. Por exemplo, se o material inicial é a boehmita, $\mathrm{AlO}(\mathrm{OH})$, formada a partir de solução de alumina amorfa, então a seqüência mais provável é $\gamma \rightarrow \delta \rightarrow \theta \rightarrow \alpha$. No entanto, se o material inicial é gibbsita, $\alpha-\mathrm{Al}(\mathrm{OH})_{3}$, então a seqüência pode incluir $\chi \rightarrow \tau \rightarrow \kappa \rightarrow \theta \rightarrow \alpha$ mesmo que seja formada boehmita antes da fase $\chi$. O diásporo, outro polimorfo do $\mathrm{AlO}(\mathrm{OH})$, transforma-se diretamente em $\alpha$-alumina. A formação das aluminas de transição a partir de compostos hidratados é acompanhada pelo desenvolvimento de uma microestrutura altamente porosa [3]. Diferentes rotas de síntese de pós de alumina estão sendo propostas de forma a se alcançar à fase estável da $\alpha-\mathrm{Al}_{2} \mathrm{O}_{3}$ sem o seu inerente crescimento dos cristais e assim obter um pó mais fino e mais reativo. O método Pechini para obtenção de óxidos cerâmicos, também chamado "método do complexo polimerizável", consiste na reação de um ácido hidroxicarboxílico com um álcool poliidroxílico, formando uma resina composta por um complexo polimérico, que tem a capacidade de quelar os íons de uma solução de cátions metálicos [8]. A pirólise desse complexo leva à formação de óxidos nanocristalinos. Do ponto de vista operacional, o método Pechini se mostra um método simples, de baixo custo e relativamente rápido, porém encontra algumas dificuldades do tocante à produção em larga escala e à eliminação de matéria orgânica $[9,10]$. Nos processos de síntese de alumina, uma das alternativas utilizadas como modificação do método, com o objetivo de diminuir a temperatura de formação da fase $\alpha$ é a adição de germens de cristalização (seeds) da própria fase $\alpha$, na forma de partículas com tamanho controlado [11-13]. Essas partículas servem, durante o processo de tratamento térmico da resina precursora, como sítios de baixa energia para a nucleação da $\alpha$-alumina, modificando o mecanismo de formação da fase. Dessa forma, ocorre a nucleação e o crescimento de partículas de $\alpha$-alumina sobre as partículas de seeds, e isso leva a um aumento na cinética de transformação de fase, bem como a uma redução significativa na temperatura necessária para a ocorrência desse fenômeno [14]. Alguns trabalhos reportam reduções de até $300^{\circ} \mathrm{C}$ na temperatura de formação da fase $\alpha[15,16]$. Resultados de pesquisas anteriores $[17,18]$ mostram também que uma atmosfera de tratamento térmico mais oxidante nas etapas finais de calcinação de pós cerâmicos levam a um aumento na eliminação de matéria orgânica residual, facilitando a cristalização da fase desejada a temperaturas mais baixas. Esse trabalho teve como objetivos sintetizar pós ultrafinos de $\alpha$-alumina com a adição de seeds, utilizando um método Pechini modificado, e verificar a influência de um fluxo de oxigênio passante no processo de calcinação do pó de alumina, em relação à eliminação de matéria orgânica residual e à microestrutura final do pó.

\section{MATERIAIS E MÉTODOS}

$\mathrm{Na}$ síntese das resinas precursoras utilizou-se ácido cítrico anidro $\left(\mathrm{C}_{6} \mathrm{H}_{8} \mathrm{O}_{7}\right.$, Vetec $)$ e etilenoglicol $\left(\mathrm{C}_{2} \mathrm{H}_{4}(\mathrm{OH})_{2}\right.$, Synth) como formadores da resina e o sal nitrato de alumínio nona-hidratado $\left(\mathrm{Al}\left(\mathrm{NO}_{3}\right)_{3} \cdot 9 \mathrm{H}_{2} \mathrm{O}\right.$, Vetec) como fonte de alumina. Na função de germens de cristalização foi utilizado um pó de alumina calcinada (A3000, Alcoa), com tamanho médio de partícula $0,5 \mu \mathrm{m}$. Para a síntese, dissolveu-se o nitrato de alumínio nona-hidratado no etlienoglicol, à temperatura de $60{ }^{\circ} \mathrm{C}$ sob constante agitação. Após a completa dissolução do sal, foi adicionado o ácido cítrico, utilizando a mínima quantidade possível de água para facilitar a sua dissolução. Em seguida foram adicionados os germens de cristalização. Manteve-se a mistura sob agitação por mais uma hora, mantendo a temperatura média de $80^{\circ} \mathrm{C}$, promovendo a liberação de gás castanho (derivados da decomposição térmica do nitrato de alumínio). Em relação às quantidades de cada componente, foi utilizada uma proporção molar de nitrato/ácido cítrico/ etilenoglicol de $0,5 / 1 / 4$. Foram produzidas inicialmente 4 resinas, variando-se a quantidade de germens de alumina em $0 \%, 5 \%, 10 \%$ e $15 \%$ em massa, em relação à massa prevista de óxido de alumínio a ser obtido com base na quantidade de nitrato de alumínio utilizado na resina. A fim de se verificar se havia alguma influência inicial no processo de síntese, amostras das resinas obtidas sem e com adição de seeds foram caracterizadas por espectroscopia no infravermelho (espectrofotometro Nicolet 4700 FTIR) e análise térmica diferencial / termogravimetria (equipamento TA Instruments SDT 2960), com taxa de aquecimento de $10{ }^{\circ} \mathrm{C} / \mathrm{min}$, da temperatura ambiente até $600{ }^{\circ} \mathrm{C}$. Após secagem das resinas por $24 \mathrm{~h}$ a $180{ }^{\circ} \mathrm{C}$, estas foram submetidas a um pré-tratamento térmico a $500{ }^{\circ} \mathrm{C}$ por $90 \mathrm{~min}$ (com taxa de aquecimento $4{ }^{\circ} \mathrm{C} / \mathrm{min}$ ) em mufla ao ar, para eliminação da matéria orgânica. As amostras resultantes, após esta etapa, foram desaglomeradas em almofariz de ágata e calcinadas a 600, 700, 800, 900, 1000 e $1100{ }^{\circ} \mathrm{C}$, por $90 \mathrm{~min}$ (com taxa de aquecimento $4{ }^{\circ} \mathrm{C} / \mathrm{min}$ ) de duas formas, com diferentes amostras: (a) em mufla ao ar e (b) em forno tubular com fluxo passante de $\mathrm{O}_{2}$. Após as calcinações, os pós obtidos foram desaglomerados em almofariz de ágata e então caracterizados por análise térmica diferencial/termogravimetria (equipamento TA Instr. SDT 2960), com taxa de aquecimento $10^{\circ} \mathrm{C} / \mathrm{min}$, da temperatura ambiente até $1300{ }^{\circ} \mathrm{C}$, difração de raios $\mathrm{X}$ (Shimadzu XRD-6000, com radiação $\mathrm{k}_{\alpha(\mathrm{Cu})}=1,54060 \AA$, com tensão $40 \mathrm{kV}$ e corrente $40 \mathrm{~mA}), \mathrm{d}(2 \theta) / \mathrm{dt}=2^{\circ} / \mathrm{min}, 2 \theta$ de $5^{\circ}$ a $90^{\circ}$. Foi também realizada a análise por espectroscopia no infravermelho (Nicolet 4700 FT-IR) e a microscopia eletrônica de varredura (Shimadzu SSX-550).

\section{RESULTADOS E DISCUSSÃO}

A análise por espectroscopia no infravermelho das resinas foi realizada com o objetivo de verificar se houve a reação de esterificação dos componentes da resina precursora, bem 


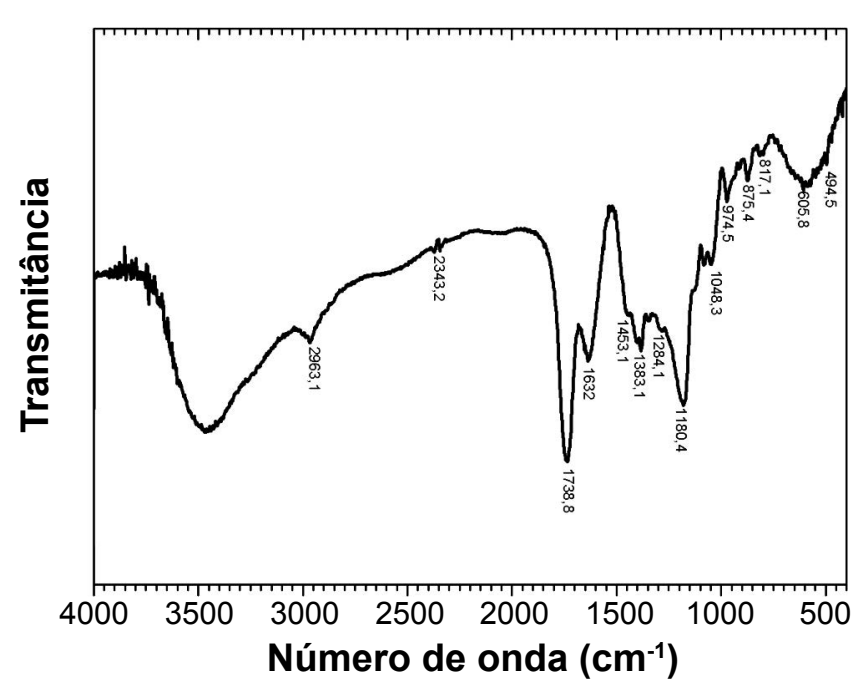

Figura 1: Espectro de infravermelho da resina após secagem em placa aquecedora, a $180^{\circ} \mathrm{C}$.

[Figure 1: Infrared spectrum of the resin after drying in heated plate, at $\left.180^{\circ} \mathrm{C}.\right]$

como se houve influência da presença de seeds na etapa inicial do processo. A Fig. 1 apresenta a espectroscopia no infravermelho da resina precursora sem seeds após secagem em placa aquecedora a $180^{\circ} \mathrm{C}$.

A banda larga observada em $\sim 3400 \mathrm{~cm}^{-1}$ corresponde ao modo de estiramento das hidroxilas, o que indica a presença de água na estrutura da resina e também adsorvida na sua superfície. Os grupos $-\mathrm{CH}_{2} \mathrm{e}-\mathrm{CH}_{3}$ presentes nos derivados orgânicos possuem banda de estiramento antissimétrico com número de onda em $\sim 2960 \mathrm{~cm}^{-1}$, bem como pela sua deformação angular localizada no espectro em $1453 \mathrm{~cm}^{-1}$. A banda em torno de $1740 \mathrm{~cm}^{-1}$ é relacionada ao estiramento do ânion carboxilato $\left(\mathrm{COO}^{-}\right)$, relacionado com a presença de complexos. $\mathrm{O} \mathrm{CO}_{2}$ presente na atmosfera é capaz de formar quelantes bidentados, cuja ligação implica em uma transferência de carga do substrato (complexo) para o adsorbato $\left(\mathrm{CO}_{2}\right)$, orientada por uma fraca doação do adsorbato para o substrato [19]. A interação do $\mathrm{CO}_{2}$ com a alumina, que recebeu um par de elétrons promove a redução do adsorbato $\left(\mathrm{CO}_{2} \rightarrow \mathrm{CO}\right)$ e, ao mesmo tempo, o substrato reoxida. O grau de hidratação da alumina, uma vez que esta tem forte afinidade com a água, é um fator influenciador nas interações do $\mathrm{CO}_{2}$ com sua superfície. A última banda claramente observada está localizada em $1630 \mathrm{~cm}^{-1}$ e pode ser associada ao modo de estiramento antissimétrico do ânion carboxilato para um complexo do tipo "ponte" [20]. A banda localizada a 1180 $\mathrm{cm}^{-1}$ caracteriza a existência de um éster originado da reação inicial entre os componentes da resina. As bandas localizadas abaixo de $1000 \mathrm{~cm}^{-1}$ correspondem às ligações primitivas de $\mathrm{Al}-\mathrm{O}$ das aluminas de transição que estão começando a se formar na amostra. A espectroscopia no infravermelho de ambas as resinas, sem e com adição de seeds, não apresentou diferença entre os espectros, constatando que os seeds não influenciam na reação de esterificação da resina precursora.

A Fig. 2 mostra a ATD/TG da resina com adição de 10\% em massa de seeds.

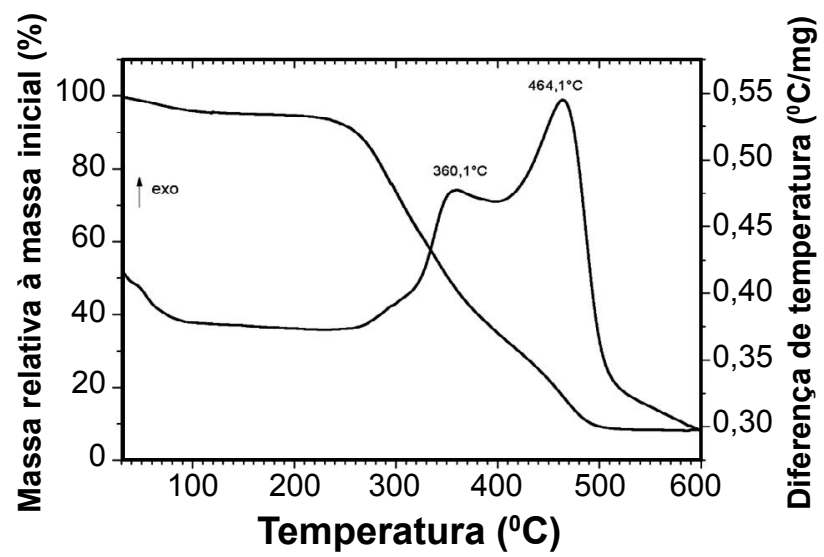

Figura 2: Curvas de análise térmica diferencial / termogravimetria da resina Pechini com adição de $10 \%$ em massa de seeds.

[Figure 2: Curves of thermal differential analysis/thermogravimetry of Pechini resin with addition of $10 \%$ weight seeds.]

$\mathrm{Na}$ termogravimetria observa-se uma perda de massa contínua que se estabiliza em torno de $10 \%$ da massa inicial e a $490{ }^{\circ} \mathrm{C}$. A primeira etapa dessa perda de massa corresponde a $10 \%$ e ocorre na faixa de temperatura de 30 a $270{ }^{\circ} \mathrm{C}$. Não se observam picos ou outras alterações relevantes no perfil de ATD nessa faixa de temperatura. Tal perda de massa refere-se à eliminação da água utilizada na síntese e à água adsorvida à resina precursora, que possui um caráter higroscópico [19]. Após $300{ }^{\circ} \mathrm{C}$, alterase a inclinação da curva de TG, denotando uma perda de massa mais vigorosa. Acompanhando tal perda de massa, observam-se duas bandas exotérmicas com seus máximos a $360{ }^{\circ} \mathrm{C}$ e a $464{ }^{\circ} \mathrm{C}$. A principal reação de decomposição se assemelha a uma reação de auto-ignição, com grande perda de massa, seguida de uma forte reação exotérmica. $\mathrm{O}$ processo de decomposição térmica das resinas Pechini comumente é composto por duas etapas, no qual uma etapa intermediária de decomposição ocorre devido à formação de um precursor estável semi-decomposto consistindo de um sal citrato complexo [19]. Esse comportamento térmico é relacionado à estabilidade do composto intermediário produzido quando os nitratos são eliminados [21]. Pelo mecanismo de reação de formação da resina precursora, a concentração de etilenoglicol é diretamente proporcional à estabilidade dos compostos intermediários, bem como ao comprimento das cadeias poliméricas. Uma vez que neste trabalho foi utilizado etilenoglicol em excesso [22], o comportamento térmico do precursor está de acordo com o que é esperado, pois a decomposição ocorreu em duas etapas facilmente distinguíveis.

A evolução das fases do material formado com a variação da temperatura de calcinação entre 500 e $1100{ }^{\circ} \mathrm{C}$ nas amostras sem seeds pode ser observada na Fig. 3.

Os pós calcinados de 500 a $700{ }^{\circ} \mathrm{C}$ são praticamente amorfos, mas de $800{ }^{\circ} \mathrm{C}$ a $900{ }^{\circ} \mathrm{C}$ os pós já apresentam algumas aluminas de transição, principalmente a fase $\gamma$. Nessa amostra também foram identificados picos menos significativos referentes às fases $\delta$ e $\theta$, com um início de cristalização da fase $\alpha$ na amostra calcinada a $900{ }^{\circ} \mathrm{C}$. Na 


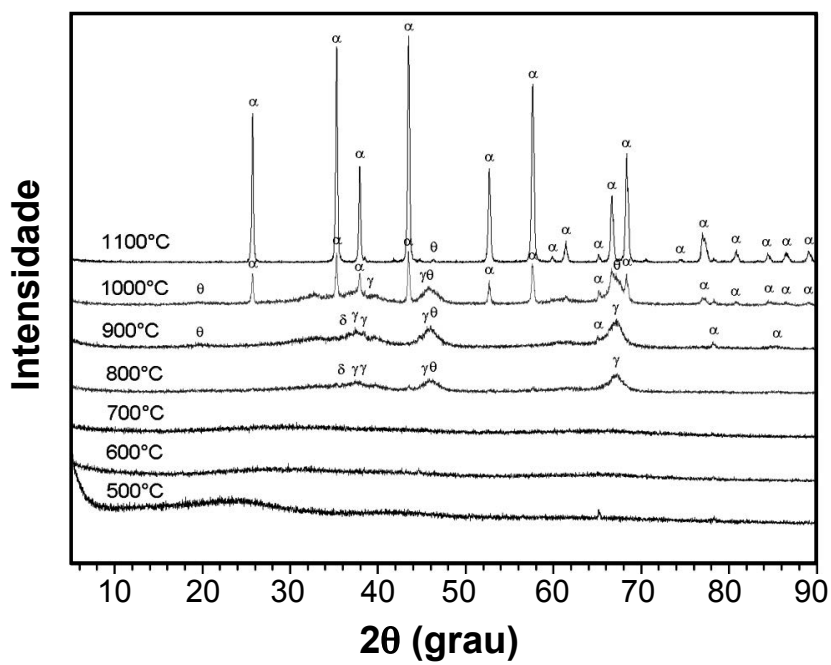

Figura 3: Difratogramas de raios $\mathrm{X}$ dos pós sem seeds calcinados de 500 a $1100{ }^{\circ} \mathrm{C}$.

[Figure 3: X-ray diffraction patterns of the powders without seeding calcined from 500 to $1100{ }^{\circ} \mathrm{C}$.]

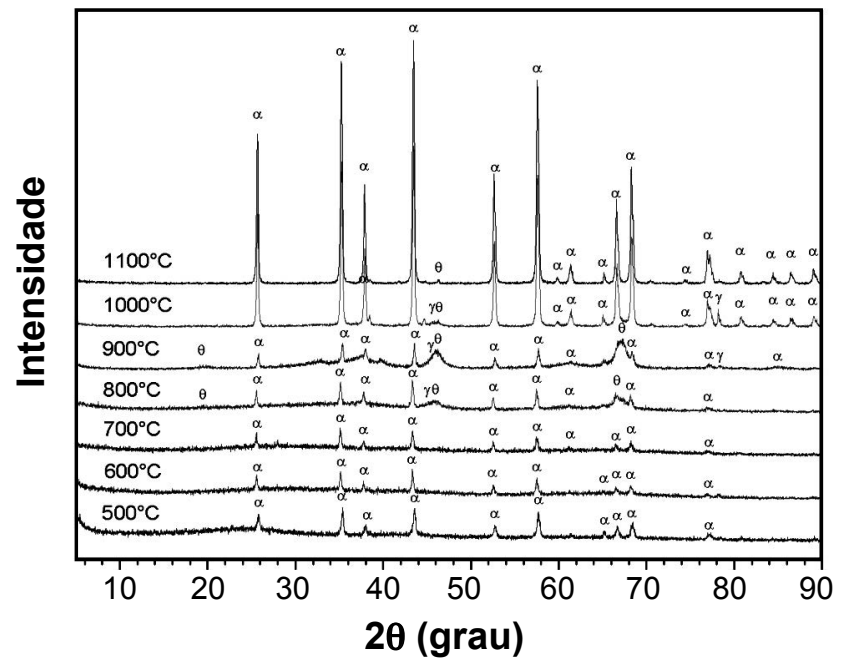

Figura 4: Difratogramas de raios $\mathrm{X}$ dos pós com seeds calcinados de 500 a $1100{ }^{\circ} \mathrm{C}$.

[Figure 4: X-ray diffraction patterns of the powders with seeding calcined from 500 to $1100{ }^{\circ} \mathrm{C}$.]

temperatura de $1000^{\circ} \mathrm{C}$, o perfil de difração se torna bastante diferente, com a maioria dos picos da fase $\alpha$ com intensidade relativamente alta, bem como ainda permanecem alguns picos das fases de transição. Também se observa uma intensidade maior nos picos da fase $\theta$, indicando que está ocorrendo uma transição $\gamma \rightarrow \theta$ na fração de alumina que ainda não se estabilizou na fase $\alpha$. A $1100{ }^{\circ} \mathrm{C}$, os picos referentes à fase $\alpha$ aumentam mais ainda sua intensidade $\mathrm{e}$ ficam mais estreitos, indicando que a maior parte da alumina de transição residual se transformou em fase $\alpha$, assim como ocorreu o crescimento das partículas.

Comparando-se com a Fig. 4, em que se têm os difratogramas de raios $\mathrm{X}$ dos pós com adição de $10 \%$ de seeds, as difrações dos pós calcinados a 500,600 e $700{ }^{\circ} \mathrm{C}$, $800{ }^{\circ} \mathrm{C}$ e $900{ }^{\circ} \mathrm{C}$, em particular, mostram alguns picos de baixa intensidade referentes apenas à fase $\alpha$, sendo esses picos correspondentes aos próprios seeds adicionados na resina. Nas amostras calcinadas a 800 e $900{ }^{\circ} \mathrm{C}$ também começam a surgir alguns picos das fases $\gamma$ e $\theta$ mais largos, característicos de pós mais finos, inerentes a essas fases. Uma redução na temperatura de formação de parte da fase $\alpha$ pode ser detectada pela melhor definição dos picos dessa fase nas amostras calcinadas a 1000 e a $1100^{\circ} \mathrm{C}$. Na amostra de $1100{ }^{\circ} \mathrm{C}$, quase não se observa nenhum pico de difração que não corresponda ao arranjo atômico dessa fase, indicando uma transformação praticamente completa.

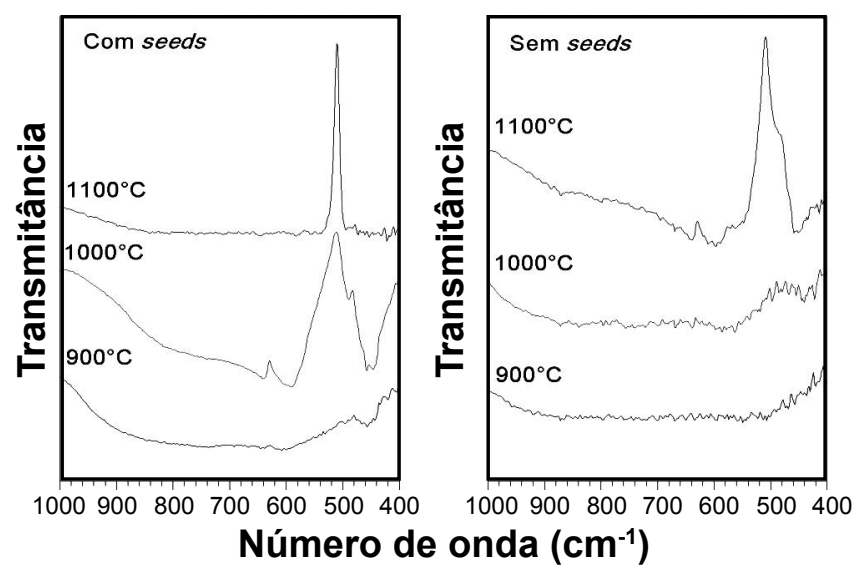

Figura 5: Espectros de infravermelho das amostras calcinadas ao ar na faixa de número de onda $400-1000 \mathrm{~cm}^{-1}$.

[Figure 5: Infrared spectra of the calcined samples under air in the wavenumber range $400-1000 \mathrm{~cm}^{-1}$.]

Por meio das seqüências de transformações observadas para todas as amostras, independente da adição de seeds, a seqüência de transformação de fases destes pós obtidos pelo método Pechini é semelhante àquela de quando o precursor é a boehmita: $\gamma \rightarrow \delta \rightarrow \theta \rightarrow \alpha$-alumina [3,23].

Para contribuir na comprovação da formação da fase $\alpha$ nas temperaturas mostradas pela análise de difração de raios X, a Fig. 5 mostra a espectroscopia no infravermelho dos pós calcinados sem e com adição de seeds na faixa de temperatura de 900 a $1100{ }^{\circ} \mathrm{C}$, na região do espectro que compreende as vibrações características dos óxidos (400 a $\left.1000 \mathrm{~cm}^{-1}\right)$.

Observam-se diferenças significativas nos espectros variando-se a temperatura de calcinação. Ocorreu um aumento na intensidade das bandas referentes às ligações octaedrais Al-O, que são características da $\alpha$-alumina, a partir das amostras calcinadas a $1000{ }^{\circ} \mathrm{C}$, principalmente na amostra em que houve adição de seeds. Apesar de ser uma análise qualitativa, pode-se dizer que o aumento da intensidade indica que porções maiores dessas amostras já se transformaram para a fase $\alpha$.

Existem duas teorias relativas ao mecanismo de nucleação da fase $\alpha$ a partir da fase $\theta$. A primeira é a teoria da nucleação por cisalhamento. Nesta teoria, sugere-se que os cristalitos de $\alpha$-alumina são "sinterizados" até um tamanho crítico, e depois o "cluster" se transforma em $\alpha$-alumina por deslocamento por cisalhamento das camadas de oxigênio. $\mathrm{O}$ 
tamanho crítico da $\theta$-alumina pode variar de $17 \mathrm{~nm}$ até várias dezenas de nm [24]. A segunda teoria é chamada de teoria da nucleação difusional. Tal teoria é baseada principalmente no efeito da adição de seeds, que trabalham efetivamente como "germens de cristalização" A principal função dos seeds é atuar como um "catalisador" da transformação de fase, diminuindo a temperatura de formação da $\alpha$-alumina ao prover sítios de baixa energia para nucleação e crescimento por difusão.

As teorias existentes são antagônicas, e a teoria da difusão por cisalhamento é fortemente rebatida em trabalhos [25], onde foi estudado o papel do "tamanho crítico" na nucleação difusional e concluiu que o "tamanho crítico" não é um prérequisito para a nucleação da $\alpha$-alumina, mas em primeiro lugar um resultado do tempo de incubação requerido para produzir núcleos de $\alpha$-alumina por nucleação difusional. O mesmo autor também sugere que os núcleos grandes de $\alpha$-alumina reportados não são devidos ao cisalhamento de cristalitos grandes de $\theta$-alumina, e sim às dificuldades de se detectarem núcleos pequenos e ao rápido crescimento da $\alpha$-alumina após a sua nucleação.

No entanto, observa-se que, nas amostras em que foram adicionados seeds, mesmo antes da nucleação da fase $\alpha$, observaram-se reflexões de fases de transição sendo formadas. Isto mostra que a fase $\alpha$ se desenvolve de duas formas independentes: a partir das fases de transição e a partir dos sítios de baixa energia formados pelas partículas de seeds previamente adicionadas ao meio, tornando-se muito mais significativa a nucleação e crescimento das partículas mesmo com uma quantidade menor de energia, isto é, a uma temperatura mais baixa.

A morfologia dos pós calcinados obtidos para todas as temperaturas de calcinação, sem e com adição de seeds, é muito semelhante. Os pós têm majoritariamente partículas grandes, que na realidade são aglomerados fortes de partículas primárias. Além disso, tentativas de sinterização desses pós mostraram que não houve densificação, evidenciando de

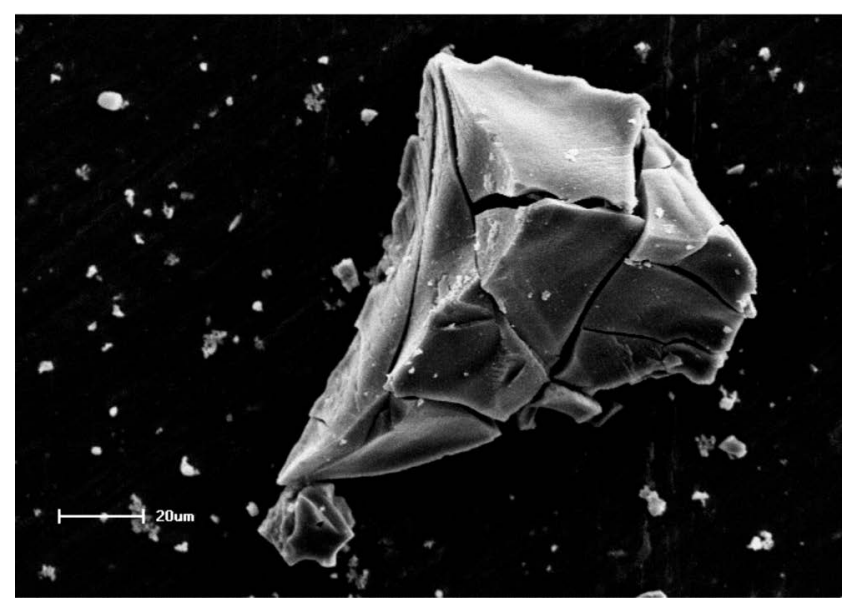

Figura 6: Micrografia obtida em microscópio eletrônico de varredura de um aglomerado de formato irregular na amostra calcinada a $1100{ }^{\circ} \mathrm{C}$ sem adição de seeds.

[Figure 6: SEM micrograph of an agglomerate of irregular shape in the sample calcined at $1100{ }^{\circ} \mathrm{C}$ without seeding.] forma direta a formação de aglomerados fortes.

A Fig. 6 mostra uma micrografia desses aglomerados fortes na amostra calcinada a $1100{ }^{\circ} \mathrm{C}$ sem adição de seeds.

Esses agregados têm formatos irregulares e uma
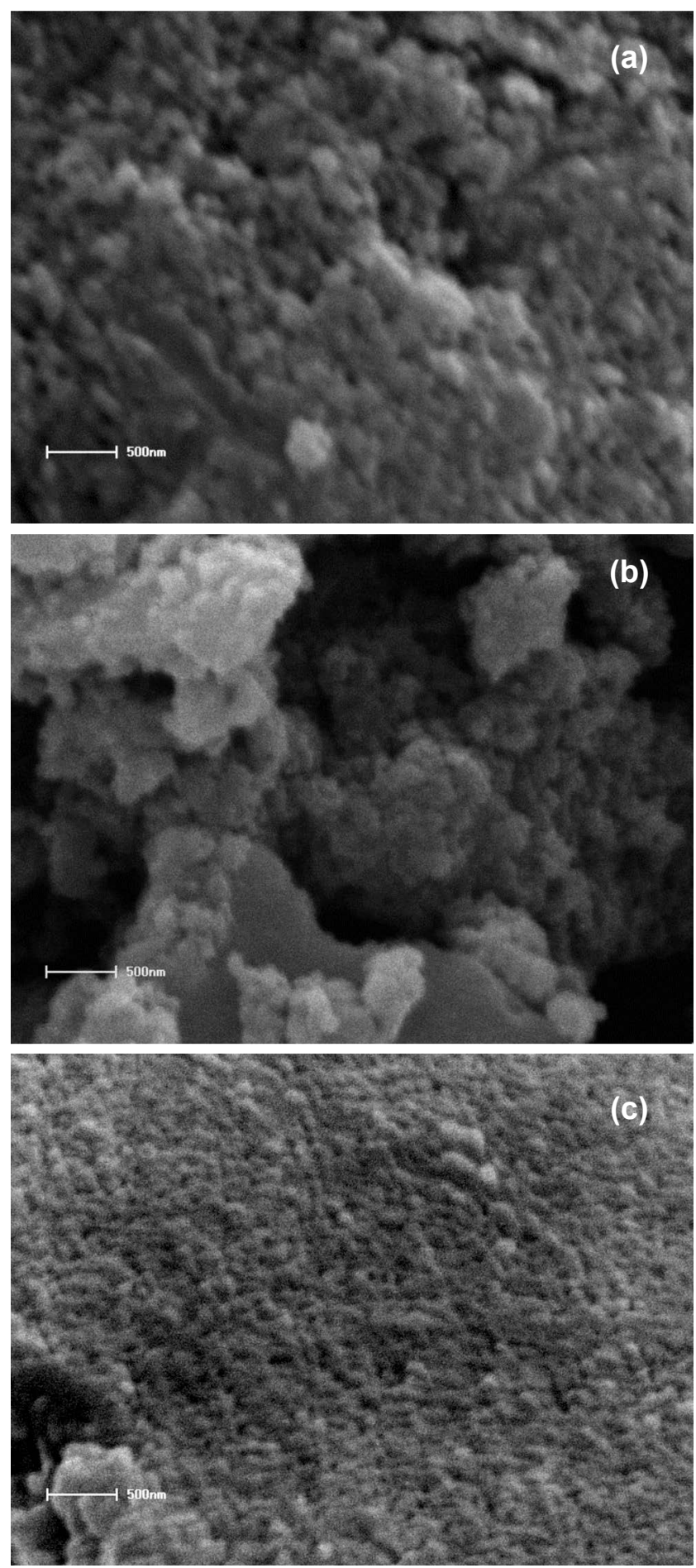

Figura 7: Micrografias obtidas em microscópio eletrônico de varredura das amostras sem adição de seeds com presença de partículas primárias: (a) amostra calcinada a $900{ }^{\circ} \mathrm{C}$; (b) amostra calcinada a $1000^{\circ} \mathrm{C}$; (c) amostra calcinada a $1100^{\circ} \mathrm{C}$.

[Figure 7: SEM micrographs of the samples without seeding with presence of primary particles: (a) sample calcined at $900{ }^{\circ} \mathrm{C}$; (b) sample calcined at $1000^{\circ} \mathrm{C}$; (c) sample calcined at $1100^{\circ} \mathrm{C}$.] 

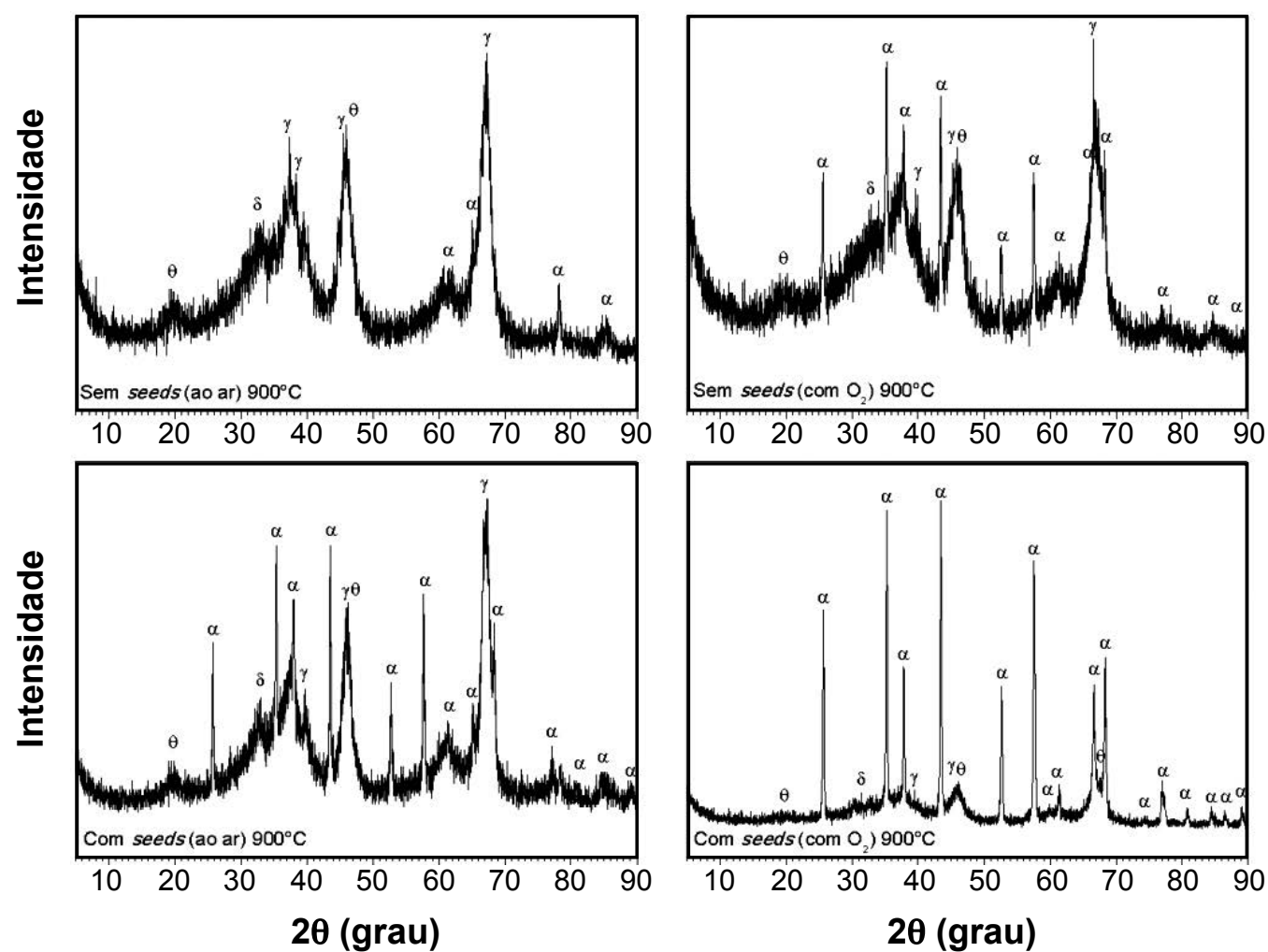

Figura 8: Difratogramas de raios $\mathrm{X}$ dos pós de alumina calcinados a $900{ }^{\circ} \mathrm{C}$.

[Figure 8: X-ray diffraction patterns of alumina powders calcined at $900^{\circ} \mathrm{C}$.]
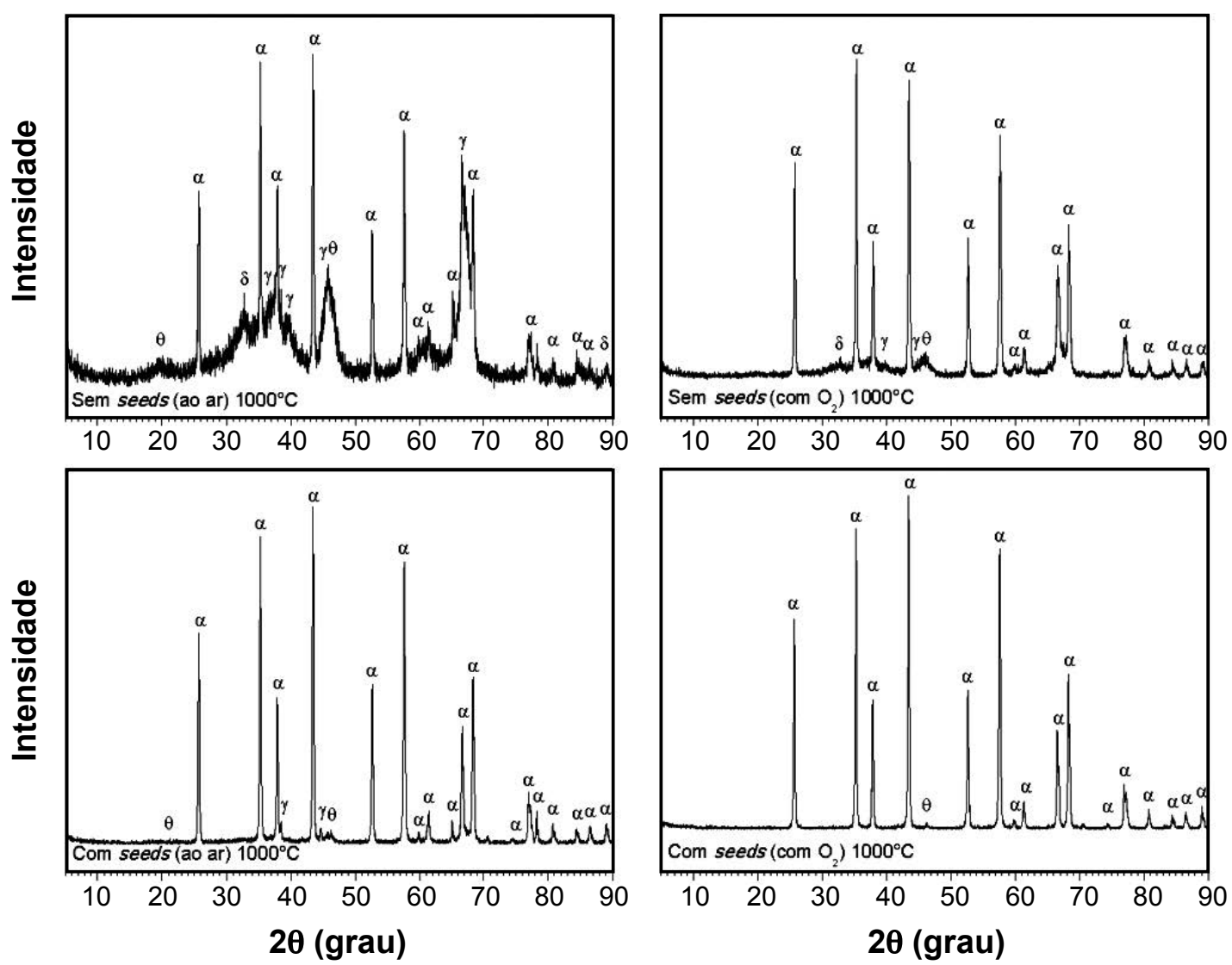

Figura 9: Difratogramas de raios $\mathrm{X}$ dos pós de alumina calcinados a $1000{ }^{\circ} \mathrm{C}$.

[Figure 9: X-ray diffraction patterns of alumina powders calcined at $1000^{\circ} \mathrm{C}$.] 

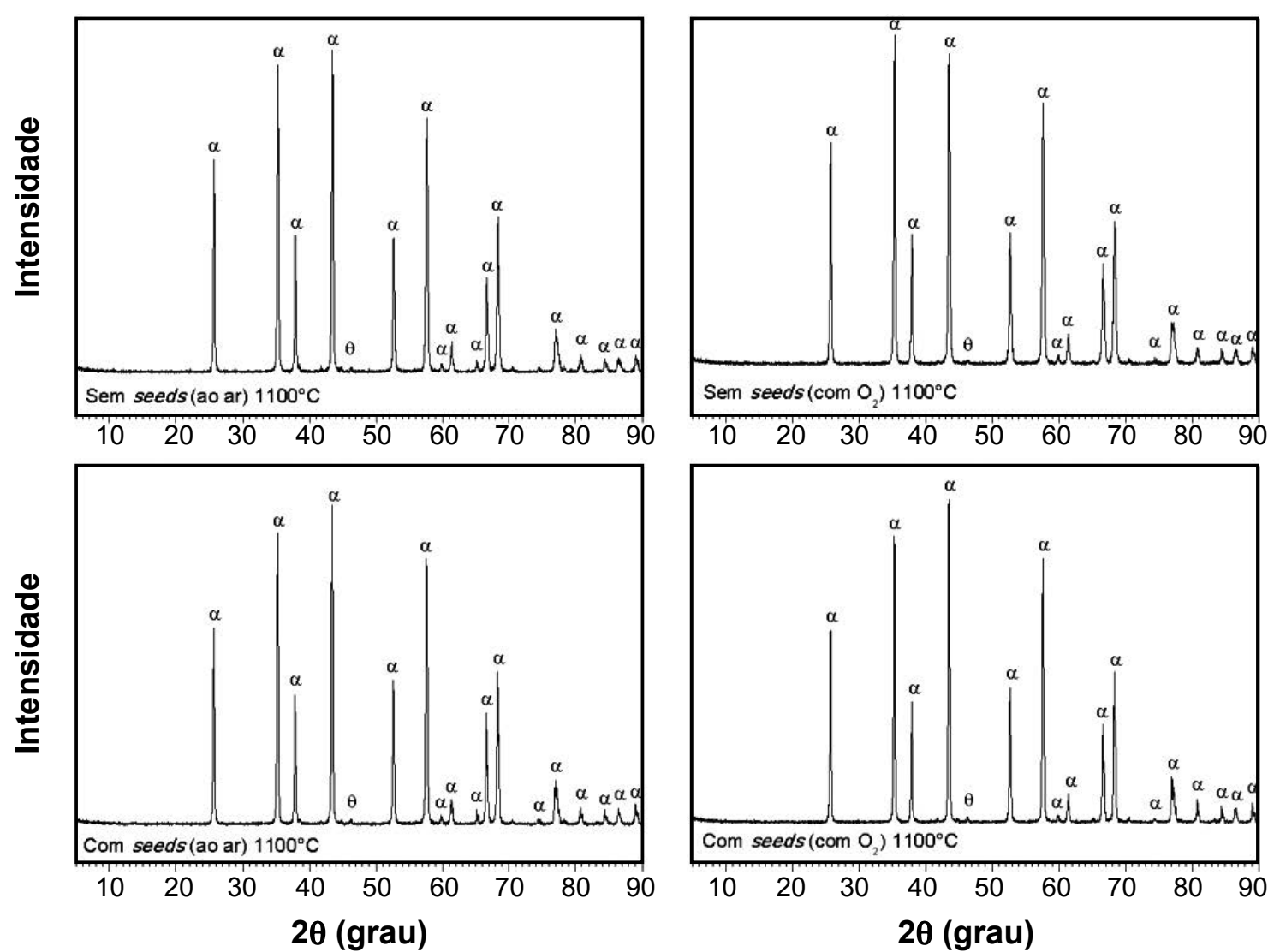

Figura 10: Difratogramas de raios $\mathrm{X}$ dos pós de alumina calcinados a $1100{ }^{\circ} \mathrm{C}$.

[Figure 10: X-ray diffraction patterns of alumina powders calcined at $1100^{\circ} \mathrm{C}$.]

superfície aparentemente lisa. Quando rompidos, tenha sido no próprio processo de síntese, ou na preparação da amostra com ultrassom, o interior desses agregados de superfície lisa revela a presença de partículas primárias de formato regular, arredondadas, e de tamanho submicrométrico, em alguns casos chegando a ser menores do que $100 \mathrm{~nm}$. A Fig. 7 mostra regiões com presença dessas partículas em interiores de agregados irregulares nas amostras calcinadas a $900{ }^{\circ} \mathrm{C}$, $1000{ }^{\circ} \mathrm{C}$ e $1100{ }^{\circ} \mathrm{C}$ sem adição de seeds.

Em um aspecto geral, através da microscopia eletrônica de varredura, as amostras com adição de seeds não apresentam muita diferença morfológica em relação às amostras sem seeds.

Os pós calcinados com fluxo de oxigênio passante em forno tubular foram primeiramente tratados em mufla ao ar a $500{ }^{\circ} \mathrm{C}$ por $90 \mathrm{~min}$, devido à grande quantidade de matéria orgânica e às reações fortemente exotérmicas ocorrentes nessa etapa da decomposição da resina.

A Fig. 8 compara os difratogramas de raios $\mathrm{X}$ dos pós de alumina calcinados em ar e em fluxo de oxigênio a $900{ }^{\circ} \mathrm{C}$ com e sem adição de seeds.

Na temperatura de calcinação de $900^{\circ} \mathrm{C}$, os pós calcinados ao ar sem seeds e com seeds mostraram um ruído bastante significativo, e os picos de maior intensidade são referentes à fase $\gamma$-alumina, largos e de baixa intensidade, o que denota a presença de pós com pequeno tamanho de partícula e baixo grau de cristalinidade. No entanto, na amostra calcinada ao ar com seeds também se observam os picos de $\alpha$-alumina que são provenientes dos próprios seeds. O perfil de difração de raios $\mathrm{X}$ do pó calcinado sem seeds em fluxo de $\mathrm{O}_{2}$ é muito semelhante ao do pó calcinado com seeds ao ar na mesma temperatura, mostrando, além dos picos da fase $\gamma$-alumina, os principais picos de difração da fase $\alpha$, o que evidencia sua existência. Pode-se, portanto, estimar que uma porção mais significativa do pó calcinado com fluxo de oxigênio já se transformou em fase $\alpha$-alumina a $90{ }^{\circ} \mathrm{C}$, em relação ao pó calcinado ao ar. $\mathrm{O}$ mesmo fenômeno ocorreu nas temperaturas de 1000 e $1100{ }^{\circ} \mathrm{C}$, em que o fluxo de oxigênio foi muito importante para favorecer a formação da fase $\alpha$, aumentando a intensidade dos picos dessa fase, como pode ser observado nos difratogramas de raios X das Figs. 9 e 10.

Apesar de ter sido grande a diferença entre as amostras sem seeds calcinadas nas duas diferentes atmosferas, entre as amostras com seeds, foi pequena a diferença qualitativa dos perfis de difração. A temperatura de $1100{ }^{\circ} \mathrm{C}$ foi suficiente para atingir uma transformação quase completa na fase $\alpha$ em todas as amostras, com um significativo grau de cristalinidade, o que pode ser determinado pela intensidade dos picos. Neste caso, o fluxo de $\mathrm{O}_{2}$ não se destacou em relação ao favorecimento da formação da fase $\alpha$, pois esta já aconteceu em temperatura menor.

A morfologia dos pós calcinados a $1000{ }^{\circ} \mathrm{C}$ com fluxo de $\mathrm{O}_{2}$ visualizados por microscopia eletrônica de varredura é apresentada na Fig. 11. Tanto o formato como 

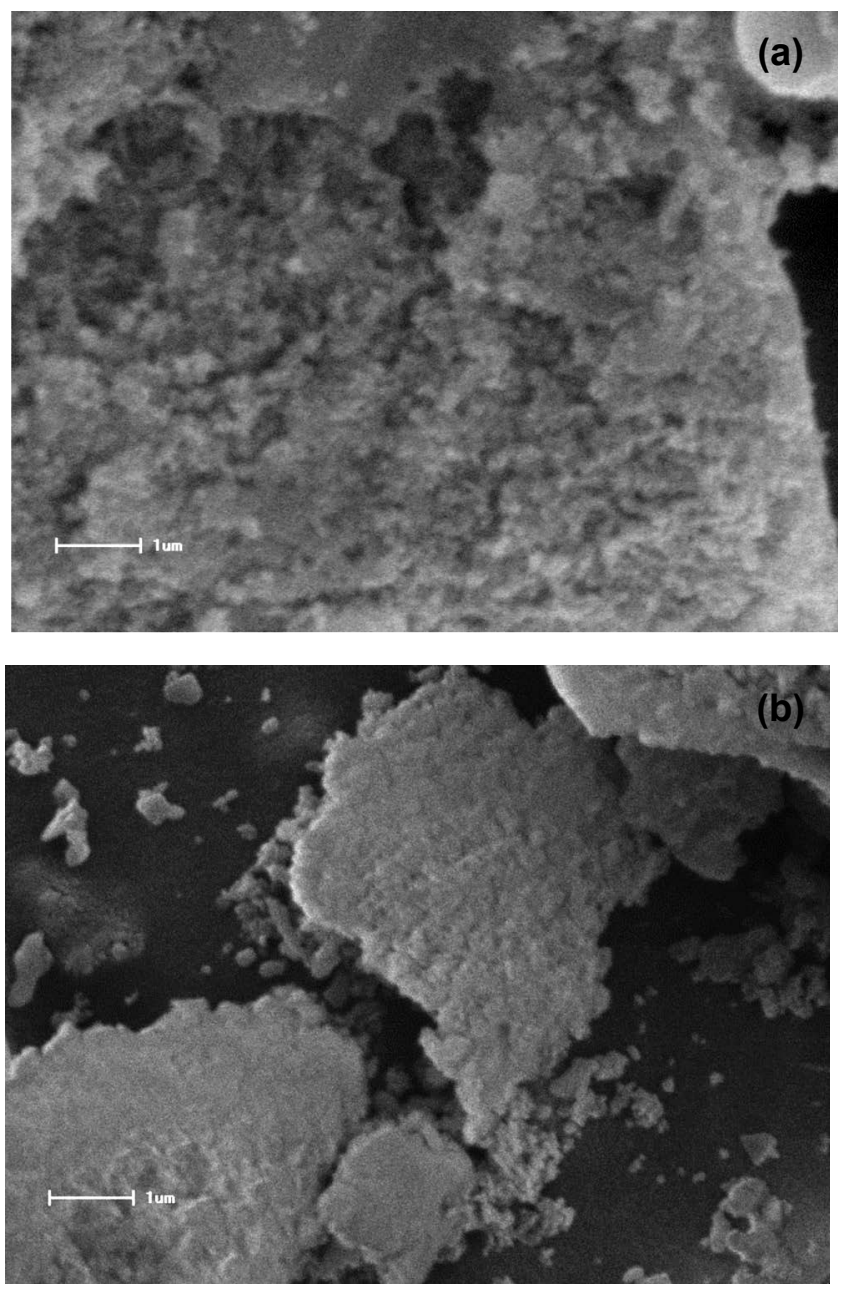

Figura 11: Micrografias obtidas em microscópio eletrônico de varredura das amostras calcinadas com fluxo de $\mathrm{O}_{2}$ à temperatura de $1000^{\circ} \mathrm{C}$ : (a) sem seeds; (b) com seeds.

[Figure 11: SEM micrographs of calcined samples with $\mathrm{O}_{2}$ flow at the temperature of $1000{ }^{\circ} \mathrm{C}$ : (a) without seeds; (b) with seeds.]

a característica dos pós não são muito diferentes das micrografias apresentadas dos pós calcinados ao ar.

Os pós são compostos por aglomerados fortes (agregados) de partículas primárias inferiores a $100 \mathrm{~nm}$ de diâmetro, não havendo uma diferença significativa entre os pós sem e com adição de seeds. Com base nos resultados expostos acima, pode-se concluir que o oxigênio contribui positivamente para a evolução das fases da alumina, formando a fase $\alpha$ a temperaturas mais baixas de calcinação mesmo sem a adição de seeds, pelo fato de contribuir para a eliminação de material orgânico. Por outro lado, a temperaturas mais altas de calcinação, a ação de uma atmosfera mais oxidante do que o ar é menos percebida, pois a existência dos seeds por si só já é suficiente para a transformação quase completa em $\alpha$-alumina. No caso em que os pós foram calcinados ao ar, os seeds exerceram um papel fundamental na redução da temperatura da fase $\alpha$. A análise morfológica dos pós não demonstrou diferenças significativas em função da variação na temperatura ou atmosfera de calcinação, sendo todas as amostras aglomerados fortes (agregados) de partículas primárias com a superfície bem consolidada, assemelhandose a uma superfície lisa.

\section{CONCLUSÃO}

A adição de sementes (seeds) no processo de síntese permitiu que a formação de $\alpha$-alumina fosse detectada a partir da temperatura de calcinação de $800{ }^{\circ} \mathrm{C}$, mas a diferença mais intensa em função da presença dos seeds é notada a $1000{ }^{\circ} \mathrm{C}$, sendo a evolução das fases de transição semelhante àquela de quando o hidróxido precursor é a boehmita. $\mathrm{O}$ fluxo de oxigênio na calcinação foi eficaz na diminuição da matéria orgânica residual, possibilitando a formação da fase $\alpha$ em quantidade significativa a $900{ }^{\circ} \mathrm{C}$. A morfologia dos pós praticamente não foi afetada pela adição de seeds e pela atmosfera com maior concentração de oxigênio, tendo sido formados aglomerados fortes de partículas primárias nanocristalinas.

\section{AGRADECIMENTOS}

Os autores agradecem a CAPES (Coordenação de Aperfeiçoamento de Pessoal em Ensino Superior) pelo apoio financeiro na realização desta pesquisa.

\section{REFERÊNCIAS}

[1] W. E. Lee, W. M. Rainforth, "Ceramic microstructures: property control by processing”, Chapman \& Hall, London, Inglaterra (1994).

[2] F. Mirjalili, M. Hasmaliza, L. C. Abdullah, Ceram. Int. 36 (2010) 1253.

[3] J. A. Thornton, J. Chin, Am. Ceram. Soc. Bull. 56 (1997) 504.

[4] A. L. Dragoo, J. J. Diamond, J. Am. Ceram. Soc. 50 (1967) 568.

[5] C. Chatfield, L. N. Lindstrom, M. E. Sjostrand, J. Phys. C 5 (1989) 377.

[6] S. J. Wilson, Proc. Brit. Ceram. Soc. 28 (1979) 281.

[7] T. C. Chou, T. G. Nieh, J. Am. Ceram. Soc. 74 (1991) 2270 .

[8] M. Pechini. US Patent 3,330,697 (1967).

[9] H. Gocmez, O. Özcan, Mater. Sci. Eng. A475 (2008) 20. [10] Z. R. Hesabi, M. Haghighatzadeh, M. Mazaheri, D. Galusek, S. K. Sadrnezhaad, J. Eur. Ceram. Soc. 29 (2009) 1371.

[11] M. Kumagai, G. L. Messing, J. Am. Ceram. Soc. 67 (1984) 230.

[12] J. L. McArdle, G. L. Messing, J. Am. Ceram. Soc. 76 (1993) 214.

[13] H. C. Kan, W. J. Wei, C. Y. Huang, J. Ceram. Proc. Res. 4 (2003) 34.

[14] Z. L. Wang, Y. Liu, Z. Zhang, "Handbook of nanophase and nanostructured materials - Synthesis", Kluwer Academic Publishers, New York, Estados Unidos (2003) passim.

[15] A. Odaka, T. Yamaguchi, T. Fujita, S. Taruta, K. Kitajima, J. Eur. Ceramic Soc. 28 (2008) 2479. 
[16] F. S. Shiau, T. T. Fang, Mater. Chem. Phys. 60 (1999) 91.

[17] H. F. Yu, Y. M. Guo, J. Alloy. Compoun. 509 (2011) 1984.

[18] A. Sinha, B. P. Sharma, H. Näfe, P. Gopalan, J. Alloy. Compoun. 502 (2010) 396.

[19] M. F. Hernández, M. González, J. Eur. Ceram. Soc. 22 (2002) 2861.

[20] S. G. Cho, P. F. Johnson, R. A. S. Condrate, J. Mater. Sci. 25 (1990) 4738.
[21] T. Zaki, K. I. Kabel, H. Hassan, Ceram. Int. 38 (2012) 2021.

[22] J. G. Li, X. Sun, Acta Mater. 48 (2000) 3103.

[23] A. Van Zyl, M. M. Thackeray, G. K. Duncan, A. I. Kingon, Mater. Res. Bull. 28 (1993) 145.

[24] X. Zhang, Y. Ge, S. Hannula, E. Levänen, T. Mäntylä, J. Mater. Chem. 19 (2009) 1915.

[25] R. B. Bagwell, G. L. Messing, P. R. Howell, J. Mater. Sci. 36 (2001) 1833.

(Rec. 27/02/2013, Ac. 12/10/2013) 